\title{
Research on Key Technology of Coal Mine Automation based on Internet of Things
}

\author{
Xueping Zhang ${ }^{1}$, Xingquan Yang ${ }^{2}$ \\ ${ }^{1}$ Research of Electronics Science and Technology Institute, Yibin University, Yibin, 644000, China \\ ${ }^{2}$ Sichuan Vocational College of Chemical Technology, Luzhou, 646000, China
}

Keywords: Internet of things. Coal mine. Automation technology

\begin{abstract}
In order to reduce coal mine accidents, control fatalities and make it easy to realize real-time monitoring and management of the personnel in wells, equipment and electric, based on present Industrial Ethernet and the technologies such as sensor for Internet of things and wireless broadband, a new network of wireless sensor and optical fiber transmission is built to reduce the hidden dangers of coal mine and accelerate the process of informatization of coal enterprises. Based on the overview of Internet of things, this paper analyzes the system configuration and characteristics of coal mine based on Internet of things, expounds the realization and structure of coal mine automation technology based on Internet of things, and demonstrates the main functions of the platform of coal mine automation technology based on Internet of things.
\end{abstract}

\section{Introduction}

Monitoring and control system of coal mine traditionally is mainly composed of control center on the ground and field control station of Inoue and the two parts, which belongs to the centralized control mode. This model has obvious shortcomings---- poor real-time performance and compatibility. In view of the development of Ethernet technology, internet in the mine begins to use high speed Ethernet, fiber communication network, computer network and embedded system and a series of advanced technologies. However, this kind of cable system has problems such as low rate, small coverage and lack of flexibility. Therefore, the author applies Internet of things combining new sensor, wireless sensor network and the RF identification technology to safety production of coal mine, thus putting forward a new development direction for safety production of modern coal mine enterprise.

\section{Overview of Internet of things}

Internet of things, as an important part of the modern information technology, refers to the Internet connecting things together. The center and foundation of Internet of things is the Internet, and is the extension and expansion in the network. Internet users can extend to reach any kind of goods and articles, implementing communication and exchange among goods. The technology of Internet of things is mainly based on the application of RFID, wireless communication technology and other means to build an overlay network, where the network communication without interference is implemented. Its essence is the automatic recognition and share of relevant information. The essence of Internet of things is the characteristic of Internet----to make sure the realization of the network of interconnection and intercommunication. Identification and communication is to have the features of automatic identification, communication and intelligentization. That is to say, the network system should possess the various functions such as automation, self feedback and intelligent control.

\section{Configuration and characteristics of coal mine automation system based on Internet of things}

The main purpose of coal mine automation system is to provide a comprehensive solution for customers so as to change the original coal mine production system, establish a monitoring system integrating multiple functions such as monitoring, equipment control and system detection, and to 
construct a monitoring platform, implementing effective monitoring of the operation of many electrical equipments and the security of coal mine in the unified network monitoring system and realizing the configuration design for the overall automation system of coal mine. Specifically, the configuration of coal mine automation system based on Internet of things has the following characteristics. The first is the high speed. In order to ensure the rapidity, stability and reliability of the processing of production data of coal mine, the automation system of coal mine provides adequate guarantee through more than two switchboards. The second is the storage. The security information of coal mine automation network system comes from the basic data of each subsystem. The real-time collection of the above security data information is done through optical fiber ring, and the information can be stored for inspection and analysis, as well as for the implementation of early warning of disasters. The third is sharing. Application of Internet of things system can fully realize the sharing of resources and enterprise customers can use Internet of things to browse and manage related data. At the same time, the enterprise managers can use Internet of things to learn the real-time production information of enterprises, which is convenient for managers to make decisions accordingly. The fourth is the safety. Internet of things for coal mine automation can use antivirus software and high-end hardware firewall for enterprise to ensure the security of Internet of things system. The fifth is stability. Internet of things system for coal mine automation adopts the structure of browser and client-site to ensure stability, high speed and smoothness of data.

\section{Approaches of coal mine automation technology based on Internet of things}

Coal mine automation technology based on Internet of things can be guaranteed by using the following access modes. The first is the OPC. Install the OPC server to achieve mutual exchange of data, use control units of working bridging and adjust communication application program to realize the storage, supply and use of real-time data. Through the design of configuration software with support for OPC protocol in the control network element server, data can be stored directly in the unit system with OPC server, and then communication program and on-site process control with the data source can be made to build bridges, exchanging real-time data with each other.

The second is the database access mode. Configuration software of Internet of things, other database and the ground control center has connection interfaces, making it possible to easily realize connection simply by corresponding configuration software.

The third is the Ethernet access model. Nowadays mine mainly uses RS485, 422 and CAN highway, whose real-time obtained data is difficult to be directly connected to switches except being converted to be transmitted to the security information server. And the application of Ethernet can make up for RS485, 422 and CAN, fostering strengths and circumventing weaknesses so as to function better. The collected real-time data can be transferred into data that supports industrial Ethernet protocol through the data transfer protocol, be transmitted to the switch, and then be processed by the acquisition server, making it possible to obtain a large amount of data information in time, improving the safety of mine. The forth is QWT198 access mode. With China's sustained economic development, the flame-proof switch which has a CAN Ethernet signal conversion appears.

\section{Structure of coal mine automation system based on Internet of things}

Internet of things for coal mine adopts comprehensive wired and wireless network structure, adding the network sensor and wireless broadband technology to the existent industrial Ethernet and then building wireless sensor and optical fiber transmission network.

In this network structure, the network system is divided into information management layer, access layer and equipment layer. A comprehensive digital information transmission platform is built based on the industrial Ethernet and fieldbus. And based on configuration software and real-time database, the mine equipment data is transmitted to a unified software platform of the dispatching control center using network technology and industrial fieldbus technology through Ethernet protocol 
to realize centralized monitoring of production equipment in the dispatching control center and improve management skills and technology level of production process.

Dagang area with a terrain that is relatively open and convenient for installing optical fiber adopts the structure of optical fiber industrial Ethernet network coupled with fieldbus network as the main network for implementation of downhole monitoring. Wireless sensor is mainly installed in rugged areas that are difficult to reach, for example, the old empty hall, wellbore and the surface of the accident prone areas. In this way the network structure can cover the entire underground region, realizing a full coverage. Embedded integrated access gateway can realize the coupling of optical fiber backbone network and wireless sensor network. Wireless sensor network and wired sensor network can transmit the collected data to the ground control center via optical fiber backbone network so that video surveillance equipment and data analysis equipment can carry out a comprehensive and real-time monitoring of the on the entire process of production. If the accident happens, the control center can direct relevant personnel and send necessary warnings according to real-time images and data obtained from the monitor. At the same time, hierarchical cluster network structure is more effective to solve the problems caused by the fact that the underground network has large area to cover, is difficult to cover and there are too many mobile nodes.

Information management layer can be divided into two parts, safety guarantee and production organization management. The relevant management personnel can use computer technology to comprehensively monitor the production status of mine to take appropriate measures in time to eliminate adverse effects and guarantee safety once abnormal situation is found. At the same time, mass data of production, organization and management can also be obtained, and through collaborative work such as production scheduling, electrical and mechanical management, logistics management and industrial automation control, work efficiency can be greatly improved.

Access layer is the core of network system, using multi fiber and multi service access platform for mine to obtain a large number of data, and through processing data by the corresponding equipment, the normal operation of network system is ensured.

Device layer mainly means that a variety of devices should operate at the same time. The work quality and performance of these devices are directly related to the accuracy for obtaining data by information management layer and the effect of safe production, organization and management of the mine. Device layer includes communication equipment, attendance positioning equipment, downhole camera, large screen, site control equipment, emergency communication equipment, ventilation equipment etc..

\section{Main function of coal mine automation platform based on Internet of things}

Information integration is the core of coal mine automation system platform based on IOT, mainly including the three-dimensional processing technique, network technique and information processing technique. With the help of the management informatization, digital image monitoring, process automation and other technology, the management of information totalization for coal mine can be done, and at the same time the demand of each production link can be satisfied, keeping the information of mine enterprise from being isolated. Specifically, the coal mine automation technology platform based on Internet of things mainly has the following five functions:

The first is to achieve safe production linkage control to realize real-time monitoring. Sensors of Internet of things in mine can collect data about humidity, temperature, gas concentration, power system state and crustal movement, etc. on the working surface, then it transmits the data to the host computer, which makes real-time display of the working state of the mine through the report, curve and 3D effect graph, etc. and immediately the give corresponding warnings before the occurrence of accidents. The host PC can depict the corresponding key regions after analyzing the real-time data so that they can carry out pertinent monitoring. In the process of roadway transportation, the winch driver can use an indicator signal to start the winch, if there is someone in its operation route, often the driver will be difficult to identify the person because of the sight line. In transportation areas there are accidents when a winch hit someone. The Internet of things is equipped with videos, audio 
sensors which can realize the real-time collection of related information of various locations such as underground yard, chamber and switch. This information, through wired relay, is transmitted to the host computer so that the ground control center of coal mine can more directly monitors the route of the winch. If the switch does not return, or someone enters the danger zone, the human body infrared sensor of Internet of things will send a signal and after the control center receives the alarm, it will immediately notifies the winch driver to correct his wrong route in time. Therefore the safety of people in transportation area is guaranteed, reducing the incidence of underground transportation accident.

The second is to achieve the tracking of personnel. The RFID reader in Internet of things is placed at the mine entrance and the main regions to comprehensively collecting information and mobile IDs of underground personnel. Its data is transmitted to the host computer through Ethernet and is stored in the database. Host PC can depict the distribution of underground personnel and track movement of the staff according to information obtained through RFID reader, and then draws corresponding statistical report forms. In this way, there is a clear grasp of the staff attendance, quantity of staff in the mine and working situation of staff, avoiding sudden situation and loss of relevant information.

The third is the realization of early warning of mine disaster. In IOT, sensor for working can collect and transmit the tunneling scale to the host computer and the forms real-time state diagrams. In light of the records of historical status of mine coal seam distribution, technical personnel can determine whether they are crossing layers or crossing the boundary. At the same time, mine experts can make plans to prevent layers crossing according to the data analysis and mark the position and the trend of layers crossing, and generate related report data to timely send out warnings of layer crossing or boundary crossing. To transmit information of seismic intensity, location and frequency to the host computer, use various networking sensor installed within the micro-seismic mining area. Technical personnel also may analyze all kinds of mining activities according to the data, and then investigate the potential threats and timely send out the warning signal in order to ensure the safe production of the mine.

The forth is the implementation of emergency rescue and disaster relief. According to the monitoring data of sensor of Internet of things, taking account of the historical data, mine experts can make corresponding emergency plans after emergency. If there is an emergency, rescue workers can quickly determine the accident location, geological structure and the best route etc. in accordance with the requirements of the plan.

The fifth is the realization of coal mine information management. That is to say, to establish the B/S mode-based mine office automation system, analysis of market coverage and coal quality, customer management, contract management, marketing management, human resource management etc..

\section{Conclusion}

Production system of coal mine is very complicated; the working environment is extremely bad; the staff is relatively concentrated; and the geological condition is changeable. Working in underground often meets new situations, for which reasonable measures are not taken in time, disasters may appear. Internet of things system for coal mine integrates the original industrial Ethernet, applies sensor technology and wireless broadband technology, and is constructed based on industrial Ethernet. This network can well meet the requirements of safety supervision, alarm and emergency processing of coal mine. It adopts office automation technology so as to truly realize many functions such as the safe production control, real-time monitoring, tracking, disaster warning and emergency and disaster relief in coal mine, which provides a good guarantee for the safe production of coal mine. 


\section{Acknowledgments}

This paper is a key science and technology project of Yibin City, Sichuan Province, code: $2011 \mathrm{GY} 028$.

\section{References}

[1] Yang Xiling. Analysis of Problems and Countermeasures in the Application of RFID Technology[J]. Coal Technology, 2011 (1)

[2] Sun Yanjing, Qian Jiansheng, Li Shiyin. Theory and Key Technology of Internet of Things System for Coal Mine[J]. Coal Science \& Technology, 2011 (2)

[3] Cheng Peng, Geng Haijiang. A Research of the Application of Internet of Things Technology in Coal Mine Management[J]. Science \& Technology Information, 2011 (9)

[4] Zhang Denghong. The Research of IOT Technology for Coal Mine Safety Inspection System[J]. Coal Technology 2011 (12)

[5] Hu Jiaying. A Study on the Relation of Internet of Things Technology and Coal Mine[J]. Science \& Technology Information, 2011 (31)

[6] Li Linchen, Yang Xiaoxue. A Design for Internet of Things System for Coal Mine Based on JN5148 [J]. Coal Technology, 2012 (12) 